\title{
The performance of derivative FTIR spectrophotometry method compared to colorimetry for tranexamic acid tablet content determination
}

\author{
Ilma Nugrahani*, Winni Nur Auli \\ School of Pharmacy, Bandung Institute of Technology \\ Jl. Ganesha 10 Bandung, Indonesia
}

Submitted: 06-12-2017

Reviewed: 24-05-2018

Accepted: 04-06-2018

\begin{abstract}
The determination of tranexamic acid levels in tablets was performed using colorimetry method in several studies. Based on its structure, tranexamic acid shows very low UV absorption which impacts to the lack of sensitivity. Then, it requires the addition of a chromophore agent to facilitate and enhance the detection limit. Recently, FTIR reported has been established as a direct content determination for some tablet dosage forms as a specific, simple, and rapid method. In this present research, the method's performance was investigated to quantify tranexamic acid in its tablet dosage form directly. The result was compared to a developed colorimetry using ninhydrin reagent. Correlations between the two methods were analyzed using t-test. The good linearity was shown at a concentration range of $0.5-1.75 \% \mathrm{w} / \mathrm{w}$ of $\mathrm{NH}$ group's spectra. Furthermore, the recovery, intra- and inter-day precision also have been proven to be fulfilled the validation requirement. Futhermore, LOD and LOQ of the method were $0.0531 \% \mathrm{w} / \mathrm{w}$ and $0.1770 \% \mathrm{w} / \mathrm{w}$ respectively. These results were next compared with the counterpart. Afterwards, the t-test statistically proved no significant difference of content determination yielded, between these two methods. In conclusion, FTIR can be used for quantifying the content of tranexamic tablet, than the colorimetry. Moreover, FTIR method also has some advantages such as easier, simpler, faster, cheaper and more sensitive than the counterpart methods. Finally, this method was suggested as a complementary for tranexamic content determination directly of its tablet dosage form.
\end{abstract}

Keywords: FTIR, derivative, quantitative, tranexamic acid, tablet, validation, colorimetry

Corresponding author:

Ilma Nugrahani

School of Pharmacy ITB,

J1. Ganesha 10 Bandung, Indonesia, 40132

Email: ilma_nugrahani@fa.itb.ac.id 


\section{INTRODUCTION}

Tranexamic acid is an antifibrinolytic drug that inhibits the breakdown of fibrin clots by blocking the binding of plasminogen and plasmin to fibrin. This drug is used for bleeding, and prophylaxis of angioedema (Sweetman, 2009). In the market, there are various forms of tranexamic acid preparations, including tablets, ampoules, vials, and capsules. Methods for testing tranexamic acid using titration are described in British Pharmacopoeia 2003, European Pharmacopoeia 4th Edition, Japan Pharmacopoeia XIV, and Chinese Pharmacopoeia. In addition, there are methods to the determination of tranexamic acid in blood and tablets using high-performance liquid chromatography (HPLC) and using TLC densitometry (Tampubolon et al., 2005).

The previous studies showed the determination of tranexamic acid levels in tablets was performed using colorimetry method (Anshari et al., 2005, Arayne et al., 2008). It was done based on the absorption of compounds in the UV-Vis region which are specific at certain wavelengths. Based on its structure, tranexamic acid has very low UV absorption and the lack of sensitivity. It requires the addition of a chromophore agent to facilitate and enhance the detection limit.

Nowadays, infrared spectroscopy commonly is a technique used for qualitative analysis, based on the atomic vibrations within a molecule. FTIR is one of it the fastest, most sensitive, and more accurate than any other. This is because the data is collected and stored in a digital form inside the computer so it can be easily changed, moved, and displayed. Analysis using FTIR was developed to overcome other methods that require complicated preparation, such as extraction and dilution. FTIR can be used for any sample form (solid, liquid, gas) directly, so that preparation will be easier (Skoog et al., 2007).

However, recently, some previous experiments showed the feasibility of FTIR for tablet's direct content determinations. The quantification method of acyclovir in single component tablet (Nugrahani and Mussadah, 2016) and vitamin B1 - vitamin B6 in a combination (Nugrahani and Kirana, 2016) has been established. The method was supported by a conversion of the absorbance spectrum into its mathematics derivative. This procedure has been improved to overcome the lack of specificity of infrared quantitative spectroscopy.

Tranexamic acid has a physical form of white and crystalline powder, soluble both in water and in glacial acetic acid. If seen from its structure, tranexamic acid has a primary $\mathrm{NH}$ group and $\mathrm{C}=\mathrm{O}$, which is rarely owned by tablet's excipients. So it was expected to produce a specific infrared spectrum of the active compound which will not be interfered by the common ingredients of the tablet matrix. Therefore, it is proper to develop a quantitative analysis using FTIR in a tablet dosage form directly.

Based on the previous information, the purpose of this study was to develop and validate a complementary to determine tranexamic acid content in tablets using FTIR. Afterwards, the result will be compared to other spectrometry. The colorimetic method has been reported using ninhydrin as the chromophore provider (Anshari et al., 2005, Arayne et al., 2008). A new method of analyzing tranexamic acid levels was hoped to deserve the better advantages.

\section{MATERIALS AND METHODS MATERIALS}

Standard of tranexamic acid (BPOM, Jakarta), tranexamic acid tablets (from the market, Bandung), matrix tablet (composed of lactose (Merck), PVP (Merck), magnesium stearate (Merck).

\section{Development of tranexamic acids FTIR method Measurement of the standard spectrum of tranexamic acid}

The method was begun with performed a variety of tranexamic acid concentrations in the $\mathrm{KBr}$ pellets. It then weighed $10 \mathrm{mg}$ of each variation and forged. After the clear pellet resulted, it was measured the AUC of a variety concentration in the range of wave numbers $4000 \mathrm{~cm}-1$ to $400 \mathrm{~cm}-1$. 
The spectrum was converted into an absorbance function of the wave number. The experiment was done triple (three times).

\section{Derivation of the standard spectrum of tranexamic acids}

The baseline in the spectrum was corrected after it was converted into an absorbance function. Then it was derivatived mathematically using the software involved in the FTIR instrument. Broadly defined AUC was formed with at some range of wave numbers.

\section{Determination of the range of FTIR number standard tranexamic acid waves}

After determination of the best wavenumber was done; a serie of tranexamic acid concentration plotted towards the AUC derivatives to find the highest linearity value.

\section{Matrix correction of wave range}

The matrix blank was made following a general formulation of tablet base. An overlay was performed between the matrix spectrum and the spectrum containing tranexamic acid.

\section{Validation of FTIR methods}

Validation method consists of specificity, linearity, detection limit, quantitation limit, range, accuracy, and precision.

\section{Specificity}

An overlay of spectra derivated was arranged between the matrix alone with the matrix mixed with active substance. The wave-numbers with the best linearity and was not influenced by matrix derivatives was selected.

\section{Linearity}

Six concentrations of tranexamic acid solutions were analyzed, and AUC measurements were made using FTIR. Then, the AUCs were plotted into concentrations ( $\% \mathrm{~b} / \mathrm{b})$. It will be accepted when $\mathrm{r}$ $\geq 0.999$ and the variance coefficient (Vxo) same or less than $2.0 \%$ (ICH, 2005).

\section{Accuracy}

The method used was additions. The tablet sample was crushed as a blank, next measured its AUC derivative using FTIR. Latest it's added with tranexamic acid in a vary concentration $(\% \mathrm{w} / \mathrm{w})$. The mixtures were blended and added with $\mathrm{KBr}$ into $100 \mathrm{mg}$. A little amount of these mixtures then were taken and pressed to make the pellets for FTIR measurements. After the spectrums were obtained, the AUC derivatives were measured minus the initial blanks. Accuracy is acceptable when recovery is $98-102 \%$ (ICH, 2005).

\section{Precision}

The method used is additions. Tablets were crushed as blanks, further measured the AUC derivatives using FTIR. Afterwards, 100\% tranexamic acid is added. The combination was blended and mixed with $\mathrm{KBr}$ to $100 \mathrm{mg}$. Measurements are repeated six times in a day and three days at different analysis times. AUC derivatives were measured for repetition of such results minus the initial blanks. Precision is acceptable if the SBR is $>2 \%(\mathrm{ICH}, 2005)$.

\section{Range}

Determination was performed by looking at the upper and lower limits of the concentration used in linearity, accuracy, and precision testing. 


\section{Limit of detection (LOD) and quantization (LOQ) \\ $\mathrm{LOD}=3(\mathrm{Sy} / \mathrm{x}) / \mathrm{b}, \mathrm{LOQ}=10(\mathrm{Sy} / \mathrm{x}) / \mathrm{b}$}

Sy / $\mathrm{x}$ = standard deviation based on the standard deviation blank, the standard deviation of the regression line, and the standard intercept y deviation on the regression line.

$\mathrm{b}=$ is the slope of the calibration curve.

\section{Sample level assessment of tranexamic acid tablets using FTIR}

Tranexamic acid tablets from the market, a total of 20 tablets, were weighed and calculated the mean. Furthermore, it is made in certain concentrations. The sample was crushed with $\mathrm{KBr}$ to $100 \mathrm{mg}$ weight. Subsequently, the AUC derivative sample was measured using FTIR and then calculated levels. The resulting content is analyzed in conformity with Indonesian pharmacopoeia requirements or other compendia requirements.

\section{Determination of sample levels tranexamate tablets use visible spectrophotometric spectrophotmetry \\ Preparation of tranexamic acid standard stock solutions}

The raw stock solution was made by dissolving $50 \mathrm{mg}$ of standard tranexamic acid in $100 \mathrm{~mL}$ of distilled water-ethanol 60:40. The solution then was sonicated for 15 minutes.

\section{Sample preparation of tablets}

Twenty tranexamic acid tablets were weighed and ground with a mortar. A total of $50 \mathrm{mg}$ of sample was transferred into a $100 \mathrm{ml}$ volumetric flask, dissolved with water-ethanol (60:40). Next it was sonicated along 15 minutes, after that added by the same solvent so that the concentration was 500 $\mu \mathrm{g} / \mathrm{mL}$. This solution was then filtered through Whatman \# 41 filter paper. Further dilution was made from this stock solution to obtain the required concentration.

\section{Derivatization for colorimetry}

A $1 \mathrm{~mL}$ of ninhydrin solution $(0.25 \% \mathrm{w} / \mathrm{v}$ in ethanol) and $1 \mathrm{~mL}$ of acetate buffer $(\mathrm{pH} 7)$ was added into the measuring flask and then added a sample of 3-25 $\mu \mathrm{g} / \mathrm{mL}$ of tranexamic acid and solvent to a limit of $10 \mathrm{~mL}$. The mixture was heated in a waterbath for 30 minutes, until a purple color was formed. The solution was cooled to room temperature, next added with ethanol to the limit marker $(10 \mathrm{~mL})$.

Maximum wavelength determination was done by making standard solution of tranexamic acid with a serie of concentration between $3-25 \mu \mathrm{g} / \mathrm{mL}$. The solutions were measured using a UV-Vis spectrophotometer at a wavelength range of $200-800 \mathrm{~nm}$, then determined the maximum wavelength (Ansari et al., 2005; Arayne et al., 2008).

\section{Verification of colorimetry methods}

The method verification consists of specificity, linearity, accuracy, precision, detection limits, and quantification limits. Specificity testing was performed by made an overlay between the matrix with the active substance spectrum. Linearity test was done by making six test solutions with variation of concentrations: $3 ; 6 ; 9 ; 12 ; 15 ; 18 \mu \mathrm{g} / \mathrm{mL}$. These were then analyzed using a UV-Vis spectrophotometer at the maximum wavelength. Triple measurements were performed. It then made a graph between the absorbance with the standard concentration of tranexamic acid and determined its linearity.

Accuracy test was done by making variation of test solution with concentration equal to 3, 9 , and $15 \mu \mathrm{g} / \mathrm{mL}$. The measured solution of each triple was next determined percent recovery rate. Precision testing was performed by making the test solutions in concentrations equal to 3,9 , and 15 
$\mu \mathrm{g} / \mathrm{mL}$ measured each on the same day, repeated in three different days, then determined the value of the coefficient variance. Limit detection and quantification limits are calculated from linearity test results.

\section{Result and discussion \\ Development of the tranexamic FTIR method}

The derivated spectrum with series concentration is shown in Figure 1. The range of wave numbers is $1511.08-1573.63 \mathrm{~cm}-1$, with the highest linearity, $\mathrm{r}=0.9994$ (Table I). The wavelength indicates the presence of the $\mathrm{NH} 2$ group on the tranexamic acid structure, and after it has been cured with a general formulation matrix, no spectra was revealed in the range of the wave numbers. So it can be concluded that the range of $1511.08-1573.63 \mathrm{~cm}-1$ (NH group) can be used as the basis for quantitative calculation of tranexamic acid level in pharmaceutical preparation.

\section{Validation of FTIR methods}

\section{Specificity}

The spectrum results should show the absence of the matrix over the specified wave number range of drug. So, this wave number area can be specified to measure the levels of tranexamic acid correctly (Figure 2).

\section{Linearity}

The measurement was conducted to the both series concentrations of $0.5-1.75 \% \mathrm{w} / \mathrm{w}$. It was determined over the wave number range $1511.08-1573.63 \mathrm{~cm}^{-1}$ (Table 2). Linear regression equation for tranexamic acid standard was resulted $\mathrm{y}=1.3568 \mathrm{x}-0.0041$ with a correlation coefficient of $(\mathrm{r})=$ 0.9994 . The value of the variance coefficient $(\mathrm{Vx} 0)$ was $1.5802 \%$. Based on these results, the values of $\mathrm{r}>0.999$ and $\mathrm{Vx} 0$ less than $2 \%$, which indicated that the method has fulfilled the linearity requirement. The graphic shown in Figure 3.

\section{Accuracy}

From the Table III, it can be seen that the resulting yield is within the required range, so this method has met the acceptance accuracy.

\section{Precision}

Intraday precision and Interday precision

Based on the Table IV and V, tranexamic acid meets the inta and inter-day precision requirements. 


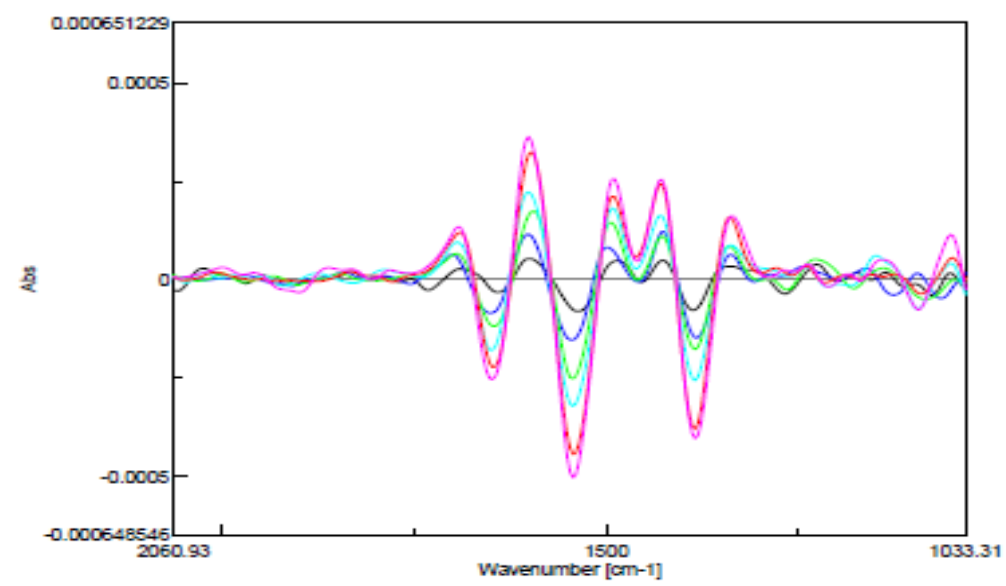

Note:

Concentration tranexamic acid standard 0,5\%

Concentration tranexamic acid standard $0,75 \%$

Concentration tranexamic acid standard 1\%

Concentration tranexamic acid standard $1,25 \%$

Concentration tranexamic acid standard $1,5 \%$

Concentration tranexamic acid standard $1,75 \%$

Figure 1. Derivated absorbance spectrum of tranexamic acid at series concentration

Figure 1 shows the linear response of the FTIR at the 3 area of wavenumbers. The ranges and values of derivated absorbance related, are listed in the Table I below.

Table I. Concentrations of tranexamic acid standard and AUC derivatives absorbance

\begin{tabular}{|c|c|c|c|c|c|c|c|}
\hline \multirow[b]{2}{*}{ Wavelength $\left(\mathrm{cm}^{-1}\right)$} & \multicolumn{6}{|c|}{ AUC at series concentration } & \multirow{2}{*}{$\begin{array}{l}\text { Coefficient } \\
\text { Corelation }\end{array}$} \\
\hline & $0.50 \%$ & $0.75 \%$ & $1 \%$ & $1.25 \%$ & $1.50 \%$ & $1.75 \%$ & \\
\hline 1511.08 & 0.0027 & 0.0045 & 0.0070 & 0.0097 & 0.0124 & 0.0152 & 0.9901 \\
\hline $1573.63-1511.08$ & 0.0030 & 0.0060 & 0.0092 & 0.0128 & 0.0163 & 0.0198 & 0.9994 \\
\hline $1573.63-1623.77$ & 0.0017 & 0.0036 & 0.0057 & 0.0074 & 0.0091 & 0.0119 & 0.9972 \\
\hline
\end{tabular}

The comparison between the wavenumber area linearity then be conducted, which the area at $1511.08-1573.63 \mathrm{~cm}^{-1}$ shows the highest $r$ (regression linear) value: 0.9994 . So, this spectra was used to further method development.

Figure 2 presents the overlay of the derivated spectrum of matrix absorbance, simulation tablets, sample tablets and tranexamic acid standard. This measurement was done to investigate whether the active compound spectrum was disturbed by other tablet's ingredient or not. Then, the figure proofs that the spectrum is not influenced by matrix's components. 


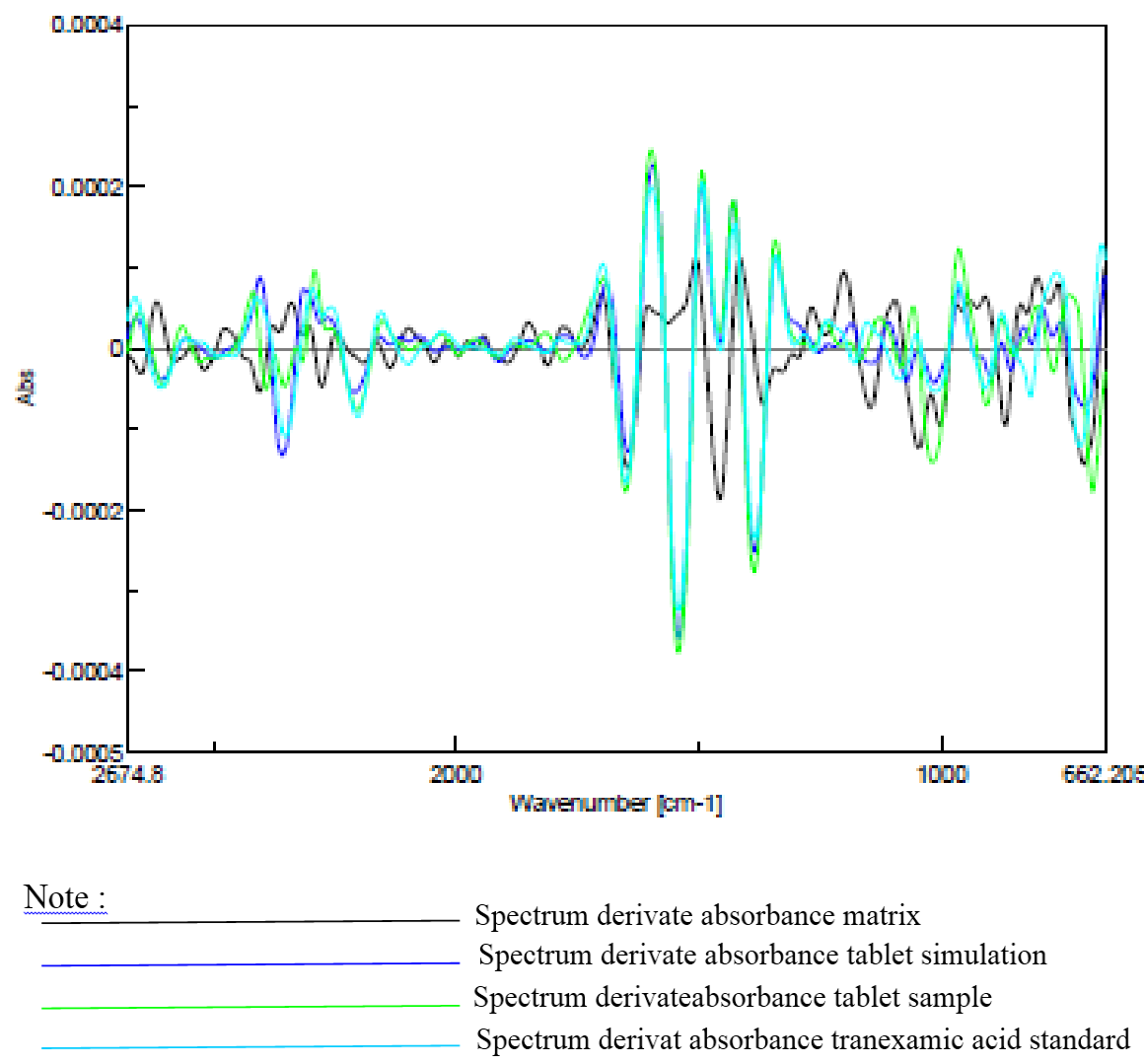

Figure 2 . The overlay of derivated spectrum of matrix absorbance, simulation tablets, sample tablets and tranexamic acid standard

Next, a calibration curve then was composed using the data obtained from a series of measurement of derivated absorbance's AUC as listed in Table II.

Table II. The series concentration series of tranexamic acid standard with AUC

\begin{tabular}{cc}
\hline $\begin{array}{c}\text { Concentration of tranexamic } \\
\text { acid (\%)/w) }\end{array}$ & $\begin{array}{c}\text { Mean AUC at wavelength } \\
\mathbf{1 5 1 1 . 0 8 - 1 5 7 3 . 6 3} \mathbf{~ m}^{-1}\end{array}$ \\
\hline 0.5 & $0.0030 \pm 0.0003$ \\
0.75 & $0.0060 \pm 0.0002$ \\
1 & $0.0092 \pm 0.0002$ \\
1.25 & $0.0128 \pm 0.0005$ \\
1.5 & $0.0163 \pm 0.0009$ \\
1.75 & $0.0198 \pm 0.0006$ \\
Linearity & 0.9994 \\
\hline
\end{tabular}

The calibration curve yielded is shown in Figure3, which show a good linearity ( $r>0.9996)$. 


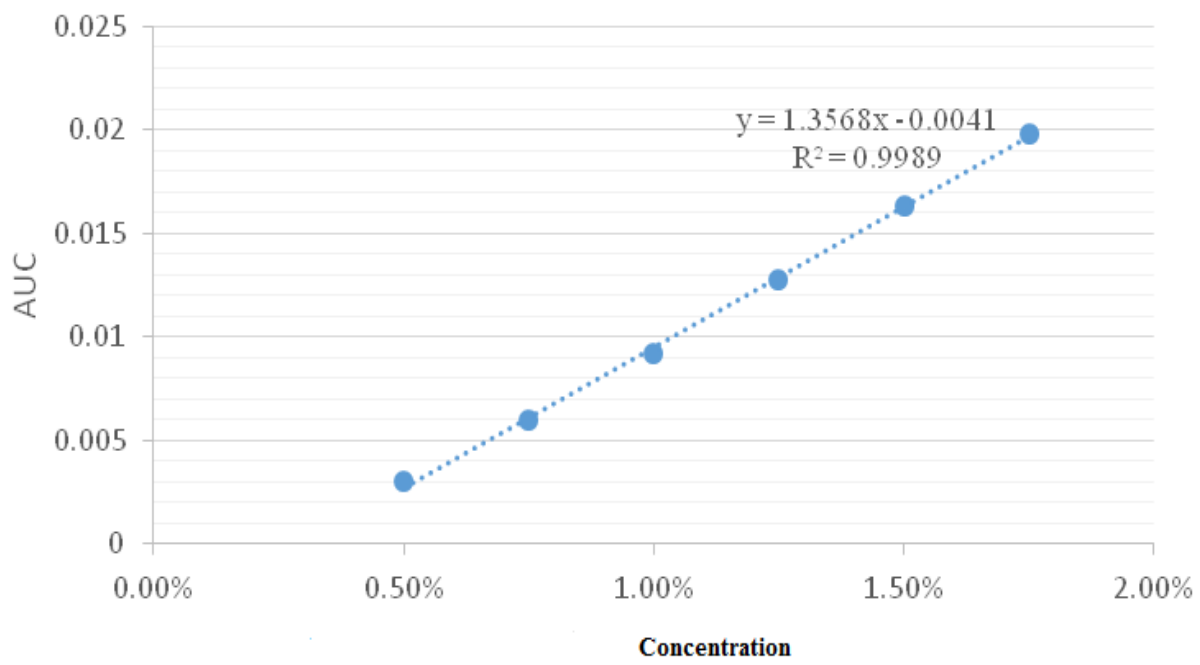

Figure 3. The linearity between concentration tranexamic acid standard to AUC derivate absorbance

Furthermore, the accuracy also has been checked to the 3 concentrations, with the result shown in Table III. The recovery values were very good and appropriated to the requirement (ICH, 2005).

Table III. Data of tranexamic acid standard accuracy test with FTIR method

\begin{tabular}{|c|c|c|c|c|}
\hline $\begin{array}{l}\text { Concentration } \\
(\%)\end{array}$ & $\begin{array}{l}\text { AUC }(1511.08- \\
\left.1573.63 \mathrm{~cm}^{-1}\right)\end{array}$ & Mean AUC & $\begin{array}{l}\text { Theoritic } \\
\text { AUC }\end{array}$ & Recovery (\%) \\
\hline 80 & $\begin{array}{l}0.0065 \\
0.0068 \\
0.0070 \\
0.0093\end{array}$ & $0.0068 \pm 0.0003$ & 0.0068 & 100.0178 \\
\hline 100 & $\begin{array}{l}0.0096 \\
0.0095 \\
0.0120\end{array}$ & $0.0095 \pm 0.0001$ & 0.0095 & 100.0130 \\
\hline 120 & $\begin{array}{l}0.0121 \\
0.0121\end{array}$ & $0.0121 \pm 0.0001$ & 0.0122 & 98.9528 \\
\hline
\end{tabular}

Intra and inter day precision test also have been performed to ensure the quality of this method, with the measurement yield shown in Table IV and V. All the data in both table supported the feasibility of the method. 
Table IV. Data of tranexamic acid standard intra-day precision test with FTIR method

\begin{tabular}{cccc}
\hline \multirow{2}{*}{ No } & \multicolumn{3}{c}{ AUC (1511.08-1573.63 cm } \\
\cline { 2 - 4 } & DAY 1 & DAY 2 & DAY 3 \\
\hline 1 & 0.0093 & 0.0092 & 0.0094 \\
2 & 0.0096 & 0.0096 & 0.0097 \\
3 & 0.0095 & 0.0093 & 0.0094 \\
4 & 0.0097 & 0.0094 & 0.0096 \\
5 & 0.0098 & 0.0093 & 0.0095 \\
6 & 0.0098 & 0.0093 & 0.0096 \\
Mean & $0.0096 \pm 0.0002$ & $0.0093 \pm 0.0001$ & $0.0095 \pm 0.0001$ \\
\%SBR & 1.9509 & 1.4942 & 1.3374 \\
\%Recovery & 101.5982 & 98.5789 & 100.7846 \\
\hline
\end{tabular}

Table V. Data of tranexamic acid standard inter-day precision test with FTIR method

\begin{tabular}{ll}
\hline Day- & AUC (1511.08-1573.63 cm $\left.\mathbf{~}^{-1}\right)$ \\
\hline 1 & $0.0096 \pm 0.0002$ \\
2 & $0.0093 \pm 0.0001$ \\
3 & $0.0095 \pm 0.0001$ \\
Mean & $0.0095 \pm 0.0001$ \\
\% SBR & 1.5579 \\
\hline
\end{tabular}

\section{Limit detection (LOD) and quantization limit (LOQ)}

Calculation of the limit detection and quantification is by the formula: LOD $=3(\mathrm{Sy} / \mathrm{x}) / \mathrm{b}=$ $0.0531 \% \mathrm{w} / \mathrm{w} ; \mathrm{LOQ}=10(\mathrm{Sy} / \mathrm{x}) / \mathrm{b}=0.1770 \% \mathrm{w} / \mathrm{w}$. Thus, the lowest detectable analytical concentration was $0.0531 \% \mathrm{w} / \mathrm{w}$, and the smallest quantitative concentration was $0.1770 \% \mathrm{w} / \mathrm{w}$.

\section{Range}

The range produces linear data and fulfill the validation acceptance test requirements with the range of $0.5-1.75 \% \mathrm{w} / \mathrm{w}$. Based on the validation test; the developed method has investigated to be fulfilled the validation requirements. Then, it can be determined to measure the tranexamic acid level of tablet dosage forms obtained from the market.

\section{Determination of tranexamic acid tablet content using FTIR}

The determination of the content is conducted by taking 20 tablet samples that have been weighted, subsequent crushed homogeneously. A few amounts of tranexamic acid tablets after crushed, was prepared to the next analysis by added with $\mathrm{KBr}$ and pressed. Six pellets yielded then were measured in FTIR. The experiment showed that the content average of the tablet was $101.503 \pm$ 0.016. The requirement of tranexamic acid levels in the tablets is $95-105 \%$ (BP, 2009), so that sample has met the criteria required by British Pharmacopeia. The data is shown in Table VI. 
Table VI. Data tranexamic acid content in tablet sample with FTIR method

\begin{tabular}{clll} 
Tablet Weight & AUC & $\begin{array}{l}\text { Mean } \\
\text { AUC }\end{array}$ & Content (\%) \\
\hline \multirow{2}{*}{$719.725 \pm 2.6103$} & 0.0094 & $0.0097 \pm$ & 101.503 \\
& 0.0103 & 0.0006 & \\
\cline { 2 - 3 } & 0.0093 & & \\
\hline
\end{tabular}

Tranexamic acid derivatization using colorimetry (UV-Vis Spectrophotometry)

FTIR method then was compared to the colorimetry which has been reported before (Anshari et al., 2005; Arayne et al., 2008). Due to the very low absorption and UV sensitivity, tranexamic acid was derivated with ninhydrin was selected as the chromophore provider reagent. In the alkaline medium, this substance converts to O-carboxyphenylglioxine, then is reduced to 2-hydroxindan-1,3dione. The primary aliphatic amino group of the drug is reacted with this reagent in a $\mathrm{pH} 7$ acetate buffer through the deamination-oxidation, which followed by the reduction of ninhydrin. The maximum color intensity will be yielded after $30 \mathrm{~min}$ heating in the waterbath. This reaction will produce Ruhemann's purple solution, which absorbs a maximum wavelength of $559 \mathrm{~nm}$ (Anshari et al., 2005; Arayne et al., 2008).

\section{Verification of the colorimetry method}

Specificity test was performed by overlaying between the matrix spectrum and the tranexamic acid standard. Based on the spectrum below, it can be seen that the presence of the matrix does not interfere with the peak of the tranexamic. Therefore, this method was set specific in the testing of tranexamic acid. The absorbance curve of the active compound is shown in Figure 4.

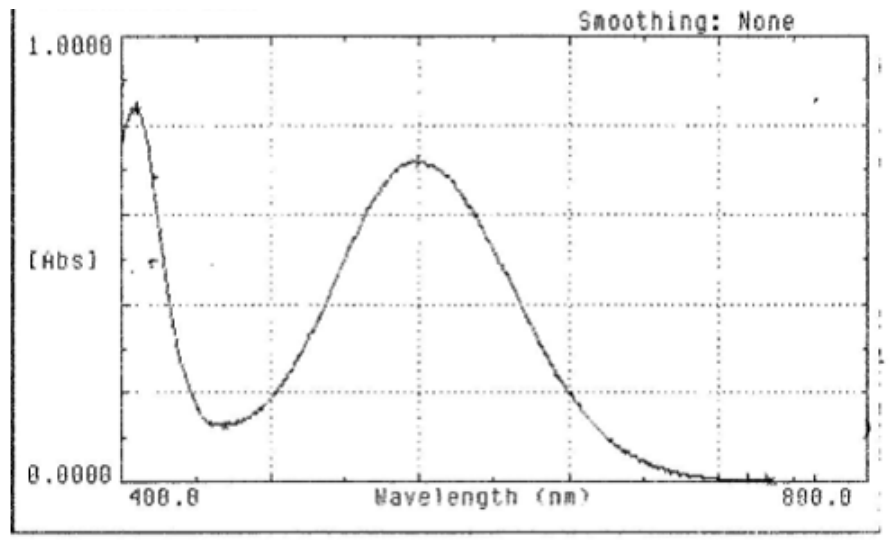

Figure 4. Overlay of spectrum tranexamic acid and matrix

Linearity was determined by measuring the absorbance of tranexamic acid derivatives at six different concentrations with a range of 3-25 $\mu \mathrm{g} / \mathrm{mL}$. The analysis was performed at a maximum wavelength of $559 \mathrm{~nm}$; each concentration was measured three times. The concentrations were plotted to absorbances. Linear regression equation yielded was $\mathrm{y}=0.0336 \mathrm{x}-0.1087$ with a correlation regression $(r)=0.9992$. The value of coefficient variance, $\mathrm{Vx} 0$, was calculated $=0.5307 \%$. Based on these results, the values of $\mathrm{r}$ was $>0.999$ and the $\mathrm{Vx} 0$ more than $2 \%$. These results indicate that the 
method has fulfilled the linearity requirement. Furthermore, the accuracy and precision, both inter- and intra-day also were checked. All the parameters showed the good result and met with requirements.

\section{Determination of tranexamic acid tablet content with colorimetry}

Twenty tablets of a tranexamic acid product were used as the sample for tested the method performance. Each tablet was weighed and then the average of 20 tablets was calculated. Next it was used to make the sample solution by coupled with ninhydrin. Furthermore, it was analyzed using spectrophotometry visible (colorimetry), which have been verified before. Then, the resulting content determination was analyzed in accordance with the requirements in the compendia. The result was $100.09 \pm 0.014 \%$, where the requirement of tranexamic acid level is $95-105 \%$ (BP, 2009). Therefore, it can be concluded that tablets have met the criteria required by British pharmacopeia.

\section{Methods comparison}

\section{Independent t-test between FTIR and colorimetry methods}

This test is applied if the data analyst aims to find out whether 2 groups of samples differ in a particular variable.

\begin{tabular}{cc} 
Table VII. T-test between FTIR and colorimetry \\
\hline \multicolumn{2}{c}{ Concentration Tranexamic Acid Tablet } \\
\hline FTIR & Colorimetry \\
99.455 & 99.636 \\
106.250 & 100.959 \\
98.806 & 98.644 \\
\hline
\end{tabular}

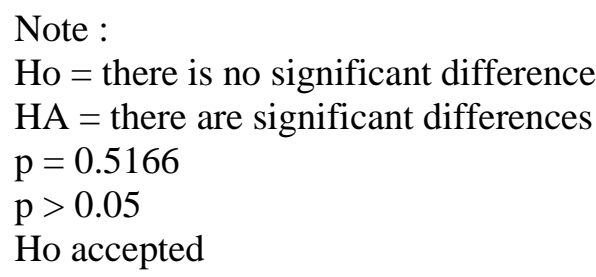

From the statistical calculation of Ho (null hypothesis), it was proved that there was no significant difference between the determination of tranexamic acid level using FTIR with the visible spectrophotometry. Furthermore, all of validation parameters such as linearity validation were compared. That were accuracy, intra day and inter day precision, timing, cost, simplicity of analysis, LOD and LOQ. From the data listed in the Table VIII, it can be seen the almost similar performance, except the sensitivity. The FTIR method has a lower sensitivity than the colorimetry, which was approximately a hundred times higher. 
Table VIII. FTIR versus colorimetry parameters

\begin{tabular}{|c|c|c|}
\hline Parameters & FTIR & Colorimetry \\
\hline Linearity (r) & 0.9994 & 0.9992 \\
\hline $\begin{array}{l}\text { Accuration/recovery } \\
(\%)\end{array}$ & $98.95-100.017 \%$ & $99.246-101.432 \%$ \\
\hline $\begin{array}{l}\text { Intraday precision } \\
(\% \text { SBR })\end{array}$ & $1.95 ; 1.49 ; 1.33 \%$ & $1.183 ; 0.884 ; 0.279 \%$ \\
\hline $\begin{array}{l}\text { Interday precision } \\
(\% \text { SBR })\end{array}$ & $1.55 \%$ & $0.239 ; 1.224 ; 1.319 \%$ \\
\hline LOD and LOQ & $\begin{array}{l}\text { LOD }=0.0531 \% \mathrm{w} / \mathrm{w} \\
(531 \mathrm{ppm}) \\
\mathrm{LOQ}=0.1770 \% \mathrm{w} / \mathrm{w} \\
(1770 \mathrm{ppm})\end{array}$ & $\begin{array}{l}\mathrm{LOD}=0.7345 \mu \mathrm{g} / \mathrm{mL}(0.7345 \\
\mathrm{ppm}) \\
\mathrm{LOQ}=2.448 \mu \mathrm{g} / \mathrm{mL}(2.448 \mathrm{ppm})\end{array}$ \\
\hline Time & 1 hour & 8 hours \\
\hline Cost & Total = IDR 130.000 & Total = IDR 366.000 \\
\hline Simplicity & Very easy and simple & $\begin{array}{l}\text { Easy but not simple due to } \\
\text { derivatitation involved heating } \\
\text { step. }\end{array}$ \\
\hline
\end{tabular}

However, the FTIR method shows the easier, simpler, faster and cheaper than its opponent. These advantages are gained from of the simplicity of sample preparation, because the extraction should not be needed. Without separation step and less solvent using, the cost and time of measurement can be reduced. On the other hand, it will be more environmental friendly. Table VIII also explains the cost of both methods, which shows that FTIR method needed third part only, compared to the colorimetry. Moreover, the time for analysis was much more reduced, until one eight from the opponent. FTIR method also showed lower sensitivity than colorimetry, which means it can measure the sample at very low concentration than colorimetry.

\section{CONCLUSION}

FTIR can be used as a newe developed method of determining tranexamic acid levels in tablets at $1511.08-1573.63 \mathrm{~cm}^{-1}$. This method has been evaluated to meet acceptance validation parameters, including specificity, linearity, accuracy, and its precision.

Conflict of Interests: There are no conflicts of interest.

\section{REFERENCES}

Ansari TM, Raza A, Rehman AU, 2005. Spectrophotometric determination of tranexamic acid in pharmaceutical bulk and dosage form, Analytical Science, 1133-1135.

Arayne MS, Sultana N, Shiddiqui FA, Mirza, AZ, Zuberi, MH, 2008. Spectrophotometric techniques to determine tranexamic acid: Kinetic studies using ninhydrin and direct measuring using ferric chloride, Journal of Melocular Structure, 891 : 475-480. 
British Pharmacopoeia, 2009. British Pharmacopoeia Volume IV, London : Medicines and Healthcare Products Regulatory Agency (MHRA), 10185.

ICH, Harmonised Tripartite Guideline, 2005, Validation of Analytical Procedures: Text and Methodology, Geneva : IFPMA.

Nugrahani I, Kirana C, 2016. Determination of thiamine $\mathrm{HCl}$ (vitamin b1) and pyridoxine $\mathrm{HCl}$ (vitamin b6) content in tablet by FTIR, International Journal Applied Pharmacy, 8 : 257-264.

Nugrahani I, Musaddah M., 2016. Development and validation analysis of acyclovir tablet content determination method using FTIR. International Journal Applied Pharmacy, 8 : 43-47.

Skoog DA, West DM, Holler FJ, 2007. Principles of Instrumenal Analysis 6th edition. Belmont: Thomson Higher Education, 430-480.

Sweetman SC, 2009, Martindale : The Complete Drug Reference 36th Edition, Chicago : Pharmaceutical Press, 552, 1080.

Tampubolon HB, Sumarlik E, Mochammad Y, Gunawan I, 2005. Densitometric determination of tranexamic acid in tablets: Validation of the Method, Journal of Liquid Chromatography and Related Technolologies, 28(20) : 3243 - 3254 . 
